\title{
Time Reversal and Its Application to Tomography with Diffracting Sources
}

\author{
Yuan Xu and Lihong V. Wang* \\ Optical Imaging Laboratory, Department of Biomedical Engineering, Texas A\&M University, \\ 3120 TAMU, College Station, Texas 77843-3120, USA \\ (Received 22 August 2003; published 23 January 2004)
}

\begin{abstract}
An exact time-domain method is proposed to time reverse a transient scalar wave using only the field measured on an arbitrary closed surface enclosing the initial source. Under certain conditions, a timereversed field can be approximated by retransmitting the measured signals in a reversed temporal order. Exact reconstruction for three-dimensional broadband diffraction tomography (a linearized inverse scattering problem) is proposed by time-reversing the measured field back to the time when each secondary source is excited. The algorithm is verified by a numerical simulation. Extension to the case using Green's function in a heterogeneous medium is discussed.
\end{abstract}

DOI: 10.1103/PhysRevLett.92.033902

Time reversal of an acoustic or electromagnetic wave is based on the invariance of the wave equation in a lossless medium under the transform $t \rightarrow-t$ ( $t$ represents the time). Time reversal of a wave can be understood as generating the back-propagation field from the measured forward-propagation field and/or its normal derivative after removing the initial sources. The concept of time reversal has been implemented experimentally and applied to a wide range of studies such as inverse scattering [1], wave-front distortion correction [2,3], and multiple scattering phenomena [4].

However, no formula is available for computing the time-reversed (TR) field using only the measured field on a closed surface enclosing the initial source. When both the field and its normal gradient on a closed surface are available, there are formulas $[3,5]$ to derive the TR field. However, it is not practical to measure both the field and its normal gradient simultaneously. For example, the output signal from a piezoelectric transducer is generally a complex combination of these two effects. There are two challenges in deriving the TR field using only the field. First, it is not obvious that Green's function, which is widely used to derive the field in space from the field on a closed surface, can be applied here. This is because the TR field on the closed surface includes both diverging and converging components $[3,5]$. While the converging component of the TR wave is just the measured signals in the forward propagation in a reversed temporal order (RTO), the diverging component has no counterpart in the forward propagation and, consequently, is not available from measurement in general. Second, in a free space, retransmission of the measured signals in RTO from the detection surface does not reproduce the TR field. This is because the waves retransmitted in one position propagate to the other positions on the surface and change the field there, and, consequently, the field on the surface does not equal the field in the forward propagation in RTO.

In this Letter, we find that when time reversal is considered in the time domain, an exact time-reversal method that uses only the field on an arbitrary closed
PACS numbers: 42.30.Wb, 42.25.Fx

surface can be derived for a wide variety of applications such as tomography with diffracting sources, inverse diffraction, and ultrasound therapy. Under certain conditions, a TR field can be approximated by retransmitting the measured signals in RTO in a free space. Acoustic waves are used as an example to present our results and methods, but their application to other scalar or vector waves is straightforward.

Next, we show that exact reconstruction for broadband diffraction tomography (DT) in a weakly scattering medium can be essentially represented by a time-reversal process in a homogeneous medium. Although exact reconstruction algorithms have been proposed for DT in some special geometries [6], no exact algorithm for broadband DT using only pressure measured on an arbitrary closed surface has been proposed. In the forward problem of our DT model, the objects [shaded in (Fig. 1)] are irradiated by an illuminating source of $\delta(t) \delta\left(\mathbf{r}-\mathbf{r}_{s}\right)$ at $\mathbf{r}_{s}$, and the scattered field is measured on an arbitrary closed surface $\Sigma$ enclosing the objects to reconstruct their heterogeneity. This DT model is a single-view one; therefore, it is very efficient for collecting data. The total field $\bar{p}_{t}(\mathbf{r}, \omega)$ in an acoustically heterogeneous medium is [7]

$$
\bar{p}_{t}(\mathbf{r}, \omega)=\bar{p}_{\text {in }}(\mathbf{r}, \omega)+\int_{R} d \mathbf{r}_{1} k^{2} \gamma\left(\mathbf{r}_{1}\right) \bar{p}_{t}\left(\mathbf{r}_{1}, \omega\right) G_{\omega}\left(\mathbf{r} \mid \mathbf{r}_{1}\right),
$$

where

$$
G_{\omega}\left(\mathbf{r} \mid \mathbf{r}_{0}\right)=\exp \left(-i \omega\left|\mathbf{r}-\mathbf{r}_{0}\right| / v_{s}\right) /\left(4 \pi\left|\mathbf{r}-\mathbf{r}_{0}\right|\right)
$$

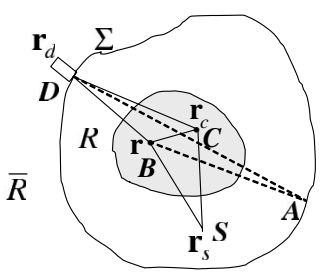

FIG. 1. Illustration of diffraction tomography. $S$ represents the illuminating source, $D$ represents a detector scanning across surface $\Sigma$, and the to-be-imaged object is shaded. $R$ and $\bar{R}$ are the spaces inside and outside $\Sigma$, respectively. 
$\bar{p}_{\text {in }}(\mathbf{r}, \omega)=G_{\omega}\left(\mathbf{r} \mid \mathbf{r}_{s}\right) / 2 \pi$ is the temporal spectrum of the incidence field, $\omega$ is the temporal angular frequency, $k=$ $\omega / v_{s}, \gamma(\mathbf{r})=\left(\kappa-\kappa_{0}\right) / \kappa_{0}$ describes the normalized deviation of compressibility $\kappa$ from the average $\kappa_{0}$, and $v_{s}$ is the acoustic speed corresponding to $\kappa_{0}$. Equation (1) can be rewritten as

$$
\bar{\varphi}_{n}(\mathbf{r}, \omega)=\int_{R} d \mathbf{r}_{1} \gamma\left(\mathbf{r}_{1}\right) \bar{p}_{t}\left(\mathbf{r}_{1}, \omega\right) G_{\omega}\left(\mathbf{r} \mid \mathbf{r}_{1}\right) / v_{s},
$$

where $\bar{\varphi}_{n}(\mathbf{r}, \omega)=\left[\bar{p}_{t}(\mathbf{r}, \omega)-\bar{p}_{\text {in }}(\mathbf{r}, \omega)\right] v_{s} / \omega^{2}$. Equation (2) shows that point $\mathbf{r}_{1}$ of the objects can be considered as a secondary source $\gamma\left(\mathbf{r}_{1}\right) \bar{p}_{t}\left(\mathbf{r}_{1}, \omega\right) / v_{s}$ for $\bar{\varphi}_{n}$. In our reconstruction of DT, we first time reverse $\bar{\varphi}_{n}$ back to time $t_{e}(\mathbf{r})=\left|\mathbf{r}-\mathbf{r}_{s}\right| / v_{s}$ at point $\mathbf{r}\left[t_{e}(\mathbf{r})\right.$ is the time the secondary source at $\mathbf{r}$ is excited] and then derive the heterogeneity. Here, we first derive the formulas for time reversing a wave in a homogeneous medium and treat DT under first Born approximation (FBA), which involves replacing $\bar{p}_{t}\left(\mathbf{r}_{1}, \omega\right)$ with $\bar{p}_{\text {in }}\left(\mathbf{r}_{1}, \omega\right)$ on the right-hand side of (2). Then the extensions to the case using Green's function in a heterogeneous medium are discussed.

We start from the wave equation for pressure $p_{0}(\mathbf{r}, t)$ in a nonabsorbing and nondispersive medium [8]

$$
\nabla^{2} p_{0}(\mathbf{r}, t)-\frac{1}{v_{s}^{2}} \frac{\partial^{2} p_{0}(\mathbf{r}, t)}{\partial t^{2}}=-q(\mathbf{r}, t)
$$

where $q(\mathbf{r}, t)$ is the source term and is nonzero only in $R$ [the space enclosed by the detection surface $\Sigma$ (Fig. 1)] and within the time period $\left[0, T_{s}\right]$. We have

$$
p_{0}(\mathbf{r}, t)=\int_{0}^{T_{s}} d t_{0} \int_{R} d \mathbf{r}_{0} q\left(\mathbf{r}_{0}, t_{0}\right) g_{+}\left(\mathbf{r}, t \mid \mathbf{r}_{0}, t_{0}\right),
$$

where $g_{ \pm}\left(\mathbf{r}, t \mid \mathbf{r}_{0}, t_{0}\right)=\delta\left(t-t_{0} \mp\left|\mathbf{r}-\mathbf{r}_{0}\right| / v_{s}\right) /\left(4 \pi\left|\mathbf{r}-\mathbf{r}_{0}\right|\right)$ is a diverging $\left(g_{+}\right)$or converging $\left(g_{-}\right)$Green's function.

Time reversal of $p_{0}(\mathbf{r}, t)$ at time $T_{0}$ is defined as

$$
p_{r}\left(\mathbf{r}, T_{0}\right)=p_{0}\left(\mathbf{r}, T_{0}\right), \quad p_{r}^{\prime}\left(\mathbf{r}, T_{0}\right)=-p_{0}^{\prime}\left(\mathbf{r}, T_{0}\right),
$$

where the prime represents the temporal derivative in this Letter, $p_{r}(\mathbf{r}, t)$ is the TR field of $p_{0}(\mathbf{r}, t)$, and $T_{0}$ is chosen to be large enough so that $p_{0}(\mathbf{r}, t)=0$ for $\mathbf{r} \in$ $R, t \geq T_{0}$. Then, $p_{r}(\mathbf{r}, t)$ can be uniquely determined by the initial conditions at $T_{0}$. The above definition of time reversal is analogous to the fact that a particle moves back along its trajectory if its velocity is reversed and its position is unchanged. According to this definition, we mean $p_{r}(\mathbf{r}, \hat{t})$ when we say time reversing a field back to time $t$, where a hat over a time variable $t$ represents $2 T_{0}-t$.

In the case of a point source $\delta\left(\mathbf{r}-\mathbf{r}_{0}\right) \delta\left(t-t_{0}\right)$ in $R$, Eq. (5) becomes $g_{r}\left(\mathbf{r}, T_{0} \mid \mathbf{r}_{0}, t_{0}\right)=g_{+}\left(\mathbf{r}, T_{0} \mid \mathbf{r}_{0}, t_{0}\right)$, $g_{r}^{\prime}\left(\mathbf{r}, T_{0} \mid \mathbf{r}_{0}, t_{0}\right)=-g_{+}^{\prime}\left(\mathbf{r}, T_{0} \mid \mathbf{r}_{0}, t_{0}\right)$. It can easily be verified that the TR field

$$
g_{r}\left(\mathbf{r}, t \mid \mathbf{r}_{0}, t_{0}\right)=g_{-}\left(\mathbf{r}, t \mid \mathbf{r}_{0}, \hat{t}_{0}\right)-g_{+}\left(\mathbf{r}, t \mid \mathbf{r}_{0}, \hat{t}_{0}\right), \quad t \geq T_{0},
$$

because it satisfies both the homogeneous wave equation and the initial-value conditions shown above. This result is also obtained by Cassereau [3] and Porter [5]. Equation (6) shows that if we time reverse at $T_{0}$, the field of a point source which is located at $\mathbf{r}_{0}$ and excited at $t_{0}$, the TR field converges to $\mathbf{r}_{0}$ at time $\hat{t}_{0}$, and then diverges with an opposite amplitude. The diverging wave $g_{+}\left(\mathbf{r}, t \mid \mathbf{r}_{0}, \hat{t}_{0}\right)$, however, does not have a counterpart in the forward propagation. It exists because, unlike the forward propagation, there is no source inside $\Sigma$ for $g_{r}(\mathbf{r}, t)$. The diverging wave with an opposite amplitude exactly cancels the source term related to the converging one.

In the case of an arbitrary source, similar results can be obtained after considering the linearity of the wave equation with respect to the source

$$
p_{r}(\mathbf{r}, t)=\int_{0}^{T_{s}} d t_{0} \int_{R} d \mathbf{r}_{0} q\left(\mathbf{r}_{0}, t_{0}\right) g_{r}\left(\mathbf{r}, t \mid \mathbf{r}_{0}, t_{0}\right) .
$$

After substituting (6) into (7), using $g_{-}\left(\mathbf{r}, t \mid \mathbf{r}_{0}, t_{0}\right)=$ $g_{+}\left(\mathbf{r},-t \mid \mathbf{r}_{0},-t_{0}\right)$, and variable transform, we have for $\mathbf{r}_{d} \in \Sigma$ (although it is valid for any $\mathbf{r}$ )

$$
\begin{gathered}
p_{r}\left(\mathbf{r}_{d}, t\right)=p_{0}\left(\mathbf{r}_{d}, 2 T_{0}-t\right)+p_{\mathrm{div}}\left(\mathbf{r}_{d}, t\right) \\
p_{\mathrm{div}}\left(\mathbf{r}_{d}, t\right)=-\int_{2 T_{0}-T_{s}}^{2 T_{0}} d t_{0} \int_{R} d \mathbf{r}_{0} q\left(\mathbf{r}_{0}, \hat{t}_{0}\right) g_{+}\left(\mathbf{r}_{d}, t \mid \mathbf{r}_{0}, t_{0}\right) .
\end{gathered}
$$

As in the case of a point source, the diverging component $p_{\text {div }}\left(\mathbf{r}_{d}, t\right)$ has no counterpart in the forward propagation and is, in general, not available from the experimental measurements of $p_{0}$ except in some special cases. Nevertheless, we show that $p_{r}(\mathbf{r}, t)$ before a specified time can be derived using only $p_{0}\left(\mathbf{r}_{d}, t\right)$.

Since there is no source for $p_{r}(\mathbf{r}, t)$ and $p_{r}\left(\mathbf{r}, T_{0}\right)=0$ in $R, p_{r}(\mathbf{r}, t)$ in $R$ can be expressed in terms of the field on $\sum[9]$ :

$$
p_{r}(\mathbf{r}, t)=-\int_{T_{0}}^{t^{+}} d t_{0} \oint_{\Sigma} d S_{d} p_{r}\left(\mathbf{r}_{d}, t_{0}\right) \frac{\partial g_{1}\left(\mathbf{r}, t \mid \mathbf{r}_{d}, t_{0}\right)}{\partial n},
$$

where $\partial / \partial n$ is the derivative along the normal of $\Sigma$ at $\mathbf{r}_{d}$ pointing away from the volume $R, g_{1}\left(\mathbf{r}, t \mid \mathbf{r}_{0}, t_{0}\right)$ with $\mathbf{r}, \mathbf{r}_{0} \in(R \cup \Sigma)$ is Green's function subject to the homogeneous Dirichlet boundary condition on $\Sigma$ [10], and $t^{+}$is infinitesimally greater than $t$. Here, we show that $p_{r o}(\mathbf{r}, t)$, the contribution of $p_{\mathrm{div}}\left(\mathbf{r}_{d}, t_{0}\right)$ to the right-hand side of (10), is zero before a specified time. After inserting (9) into (10), we have

$$
p_{r o}(\mathbf{r}, t)=\int_{T_{0}}^{t^{+}} d t_{0} \oint_{\Sigma} d S_{d} \int_{2 T_{0}-T_{s}}^{2 T_{0}} d t_{c} \int_{R} d \mathbf{r}_{c} q\left(\mathbf{r}_{c}, \hat{t}_{c}\right) g_{+}\left(\mathbf{r}_{d}, t_{0} \mid \mathbf{r}_{c}, t_{c}\right) \frac{\partial g_{1}\left(\mathbf{r}, t \mid \mathbf{r}_{d}, t_{0}\right)}{\partial n} .
$$

Considering $g_{+}\left(\mathbf{r}_{d}, t_{0} \mid \mathbf{r}_{c}, t_{c}\right)=0$ when $t_{0}<t_{c}+\left|\mathbf{r}_{c}-\mathbf{r}_{d}\right| / v_{s}$ and $g_{1}\left(\mathbf{r}, t \mid \mathbf{r}_{d}, t_{0}\right)=0$ when $t<t_{0}+\left|\mathbf{r}-\mathbf{r}_{d}\right| / v_{s}$, and 
assuming that $q\left(\mathbf{r}_{c}, t_{c}\right)$ is nonzero only within the period $\left[t_{c 1}, t_{c 2}\right]$, we conclude that $p_{r o}(\mathbf{r}, t)=0$ if for any $\mathbf{r}_{c} \in R$ and $\mathbf{r}_{d} \in \Sigma$

$$
t<2 T_{0}-t_{c 2}+\left|\mathbf{r}_{d}-\mathbf{r}_{c}\right| / v_{s}+\left|\mathbf{r}-\mathbf{r}_{d}\right| / v_{s} .
$$

Actually, $p_{r o}(\mathbf{r}, t)$ can be shown to be equivalent to the field induced by the reflection of the diverging field $p_{\text {div }}(\mathbf{r}, t)$ by $\Sigma$. It contributes only to the late part of $p_{r}(\mathbf{r}, t)$. In many applications including tomography with diffracting sources and ultrasound therapy, we are interested only in the part of $p_{r}(\mathbf{r}, t)$ within the range defined by (12). For example, in our DT model, the heterogeneity at point $\mathbf{r}$ in Fig. 1 can be derived from $p_{r}\left(\mathbf{r}, \hat{t}_{e}(\mathbf{r})\right)$, as is shown in (17). Noticing $\left|t_{e}(\mathbf{r})-t_{e}\left(\mathbf{r}_{c}\right)\right| \leq$ $\left|\mathbf{r}-\mathbf{r}_{c}\right| / v_{s}$ and $t_{c 1}=t_{c 2}=t_{e}\left(\mathbf{r}_{c}\right)$, we find that (12) is minimally observed for any $\mathbf{r}_{c} \in R$ and $\mathbf{r}_{d} \in \Sigma$ to compute $p_{r}\left(\mathbf{r}, \hat{t}_{e}(\mathbf{r})\right)$ in DT. Similarly, we find that (12) can easily be met for other applications such as thermoacoustic tomography and ultrasound therapy. Therefore, $p_{r}(\mathbf{r}, t)$, within the range defined by (12), can be expressed as

$$
p_{r}(\mathbf{r}, t)=-\int_{T_{0}}^{t^{+}} d t_{0} \oint_{\Sigma} d S_{d} p_{0}\left(\mathbf{r}_{d}, \hat{t}_{0}\right) \frac{\partial g_{1}\left(\mathbf{r}, t \mid \mathbf{r}_{d}, t_{0}\right)}{\partial n} .
$$

Equation (13) shows that the TR field before a certain time in $R$ is equivalent to the field [caused by the retransmission of $p_{0}\left(\mathbf{r}_{d}, t\right)$ from $\Sigma$ in RTO] in a reflective cavity (formed by $\Sigma$ ) rather than in a free space. Therefore, the reflections from $\Sigma$ also contribute to $p_{r}(\mathbf{r}, t)$. The reason is that the field transmitted in one position on $\Sigma$ propagates to the other positions on $\Sigma$, and, consequently, the field in these positions changes. The reflections from $\Sigma$ cancel the changes in these positions.

Next, we show that the reflections from $\Sigma$ are negligible under certain conditions. The central concept is that $g_{1}\left(\mathbf{r}, t \mid \mathbf{r}_{d}, t_{0}\right)$ can be obtained in a model where the boundary $\Sigma$ serves as a perfect mirror (with a phase shift of $\pi$ after reflection), and a delta pulse source is launched at $\mathbf{r}_{d}$ and time $t_{0}$. Under the ray approach (geometrical optics approximation), $g_{1}\left(\mathbf{r}, t \mid \mathbf{r}_{d}, t_{0}\right)$ is the summation of the contributions from all the rays that go from $\mathbf{r}_{d}$ and arrive at $\mathbf{r}$ if the radii of $\Sigma$ are much larger than the wavelength of the excitation pulse. As shown in Fig. 1, the first ray from $\mathbf{r}_{d}$ to $\mathbf{r}$ travels along the line connecting $\mathbf{r}_{d}$ and $\mathbf{r}$. The second ray is first reflected by the boundary at $\mathbf{r}_{d}$ and then goes to $\mathbf{r}$. Both of them arrive at $\mathbf{r}$ at time $t_{0}+$ $\left|\mathbf{r}-\mathbf{r}_{d}\right| / v_{s}$. The contribution of the first two rays to $\partial g_{1} / \partial n$ in (13) is $2 \partial g_{+} / \partial n$. Then there are other rays (for example, the dashed line in Fig. 1) that are reflected at other points on the boundary such as $A$. In DT, it can be shown that when the to-be-reconstructed point $\mathbf{r}$ is near the center of $\Sigma$, and $l_{\text {det }}>2 l_{\mathrm{obj}}$, where $l_{\text {det }}$ is the average linear dimension of $\Sigma$ and $l_{\mathrm{obj}}$ is the maximum linear dimension of the object, $p_{r}\left(\mathbf{r}, \hat{t}_{e}(\mathbf{r})\right)$ can be approximated well after replacing $\partial g_{1} / \partial n$ in (13) with $2 \partial g_{+} / \partial n$ [11]

$$
p_{r}(\mathbf{r}, t) \approx-2 \int_{T_{0}}^{t^{+}} d t_{0} \oint_{\Sigma} d S_{d} p_{0}\left(\mathbf{r}_{d}, \hat{t}_{0}\right) \frac{\partial g_{+}\left(\mathbf{r}, t \mid \mathbf{r}_{d}, t_{0}\right)}{\partial n}
$$

Equation (14) shows that under certain conditions, time reversal can be approximated well by retransmission of the measured signals in RTO from the detection surface in a free space. Equation (14) also holds for applications other than DT when an appropriate $l_{\text {det }}$ is chosen by using the demonstrated method for DT. Equation (14) can be transformed into

$$
\begin{aligned}
p_{r}(\mathbf{r}, t) \approx \frac{1}{2 \pi} \oint_{\Sigma} d S_{d} \frac{\mathbf{n} \cdot\left(\mathbf{r}_{d}-\mathbf{r}\right)}{\left|\mathbf{r}-\mathbf{r}_{d}\right|^{2}} & \frac{p_{0}\left(\mathbf{r}_{d}, t_{r d}\right)}{\left|\mathbf{r}-\mathbf{r}_{d}\right|} \\
& \left.-p_{0}^{\prime}\left(\mathbf{r}_{d}, t_{r d}\right) / v_{s}\right],
\end{aligned}
$$

where $t_{r d}=2 T_{0}-t+\left|\mathbf{r}-\mathbf{r}_{d}\right| / v_{s}$. Equation (15) is in the form of the well-known delay-and-sum algorithm (backprojection to spheres) used in synthetic aperture imaging. Consequently, the physical meaning and the valid conditions of this widely used algorithm are revealed quantitatively for the first time from basic physics.

Now we discuss the reconstruction in DT. In the forward propagation, the secondary sources (the points of the objects) are not excited at the same time, since the incidence wave reaches different points at different times. Therefore, in the TR field, the diverging waves from some secondary sources mingle with the converging waves from other secondary sources according to (6). Nevertheless, we show that there is a strikingly simple relationship between the TR field and the heterogeneity under FBA. After combining (2), (6), and (7) and the application of inverse Fourier transform, the TR field of $\bar{\varphi}_{n}(\mathbf{r}, \omega)$ at time $\hat{t}_{e}(\mathbf{r})$ is

$$
\begin{array}{rl}
\varphi_{n r}\left(\mathbf{r}, \hat{t}_{e}(\mathbf{r})\right)=\int_{R} & d \mathbf{r}_{1} \gamma\left(\mathbf{r}_{1}\right) \\
& \times \int_{-\infty}^{\infty} d k \exp \left(-i k\left|\mathbf{r}-\mathbf{r}_{s}\right|\right) \bar{p}_{t}\left(\mathbf{r}_{1},-\omega\right) \\
& \times\left[G_{\omega}^{*}\left(\mathbf{r} \mid \mathbf{r}_{1}\right)-G_{\omega}\left(\mathbf{r} \mid \mathbf{r}_{1}\right)\right],
\end{array}
$$

where * represents the complex conjugate. After applying FBA and some mathematical manipulations, we have

$$
\gamma(\mathbf{r})=4 \frac{\partial\left[\left|\mathbf{r}-\mathbf{r}_{s}\right| \varphi_{n r}\left(\mathbf{r}, \hat{t}_{e}(\mathbf{r})\right)\right]}{\partial\left|\mathbf{r}-\mathbf{r}_{s}\right|} .
$$

Therefore, the reconstruction in DT can be implemented by first time reversing $\varphi_{n}$ to obtain $\varphi_{n r}\left(\mathbf{r}, \hat{t}_{e}(\mathbf{r})\right)$ with (13) or (14), and then obtaining $\gamma(\mathbf{r})$ with (17).

A three-dimensional DT is numerically simulated (Fig. 2). The object is a sphere with a radius of $8 \mathrm{~mm}$ at the origin, and to validate FBA, $\gamma(\mathbf{r})$ is set to be 0.01 in the sphere and zero otherwise [12], and $v_{s}$ is $1.5 \mathrm{~km} / \mathrm{s}$ in the background. The illuminating source is at $[0,64,0] \mathrm{mm}$. The 8192 detection positions are randomly 

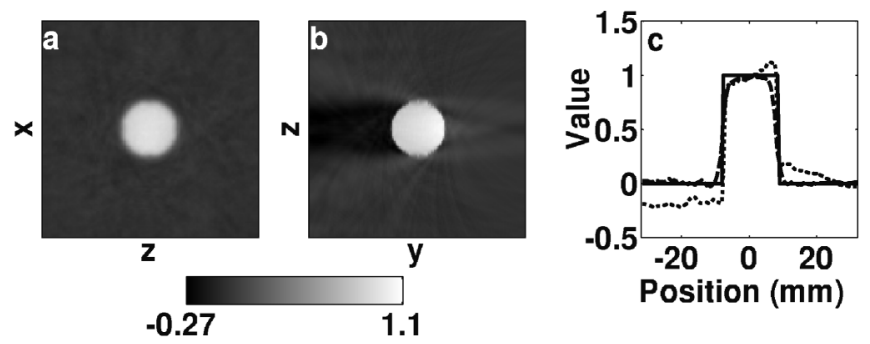

FIG. 2. (a) Reconstructed image section in DT along the $y=0$ and (b) $x=0$ planes. (c) The line graphs along the $z=0$ (dotted line), $y=0$ (dashed line) lines in (b) and the corresponding real value (solid line). The object values are normalized to 0.01 .

distributed over an ellipsoid with three axes of [80, 64, $80] \mathrm{mm}$ long and the center at the origin. The imaging space is a cube with a side length of $64 \mathrm{~mm}$, centered at the origin, and mapped with a 128 by 128 by 128 mesh. In the forward problem, $\varphi_{n}(\mathbf{r}, t)$ is computed by the integration of the object value along a series of ellipsoids. The signals are within [0 1.5] MHz. In the inverse problem, we use (15) instead of the exact formula (13) to time reverse fields to save computation time. We swap the order of (17) and the integration in (15) to improve the computation accuracy. Figures 2(a) and 2(b) show that the shape and position of the object are reconstructed correctly. The line graphs in Fig. 2(c) show that the object is reconstructed quantitatively. There are some shadows from the object along the line ( $y$ axis) connecting the source and the object in Fig. 2(b). The shadows rotate when the position of the source changes. The shadows are probably caused by the limited bandwidth of the simulated signals. They can be eliminated if the illuminating source is placed in several positions and multiple sets of data are collected.

Our time-reversal methods are derived for waves in a homogeneous medium. The extension of most of them to a heterogeneous medium is straightforward. Equations (4)(10) hold for a heterogeneous medium after Green's function is replaced by the corresponding one in a heterogeneous medium. In addition to replacing Green's function, $v_{s}$ in (12) should be understood as the maximum of the acoustic speed in the medium to extend (11)-(13). Equation (14) can be extended after the minimum requirement for $l_{\mathrm{det}}$ is estimated. This estimation is more complex in a heterogeneous medium, since Green's function in a heterogeneous medium is usually a wave train rather than a delta pulse. To proceed from (14) to (15), we need to replace $\left|\mathbf{r}-\mathbf{r}_{d}\right| / v_{s}$ in (15) with $t_{f}\left(\mathbf{r}, \mathbf{r}_{d}\right)$, the flight time from $\mathbf{r}$ to $\mathbf{r}_{d}$, and assume that the heterogeneity changes only the flight time of a transient wave.

FBA is used in our discussion about DT. We realize that FBA does not hold for high frequencies. However, if we confine the frequency response range of the ultrasound transducer to the low-frequency range, FBA stands approximately. Alternatively, we can improve FBA by applying a distorted-wave Born approximation and timereversal methods using Green's function in a heterogeneous medium. At last, it should be pointed out that our time-reversal methods can be easily applied to inverse diffraction, inverse source problems, and other tomographies using a diffracting source such as thermoacoustic tomography.

We are grateful to Minhua $\mathrm{Xu}$ for useful discussion about this study. This project was sponsored in part by U.S. Army Medical Research and Material Command Grant No. DAMD17-00-1-0455, National Institutes of Health Grant No. R01 EB000712, and Texas Higher Education Coordinating Board Grant No. ARP 000512-0063-2001.

*Email address: LWang@tamu.edu

[1] R. P. Porter, J. Acoust. Soc. Am. 80, 1220 (1986); J. Thomas, P. Roux, and M. Fink, Phys. Rev. Lett. 72, 637 (1994); J. H. Rose, in Imaging of Complex Media with Acoustic and Seismic Waves, edited by M. Fink, W. A. Kuperman, J. P. Montagner, and A. Tourin, Topics in Applied Physics Vol. 84 (Springer, Berlin, 2002), p. 97.

[2] M. Fink, IEEE Trans. Ultrason. Ferroelectr. Freq. Control 39, 555 (1992).

[3] D. Cassereau and M. Fink, IEEE Trans. Ultrason. Ferroelectr. Freq. Control 39, 579 (1992).

[4] A. Tourin, A. Derode, and M. Fink, Phys. Rev. Lett. 87, 274301 (2001)

[5] R. P. Porter, Phys. Lett. 29A, 19 (1969).

[6] E. Wolf, Opt. Commun. 1, 153 (1969); A. J. Devaney, Ultrason. Imaging 4, 336 (1982); T. Melamed, Y. Ehrlich, and E. Heyman, Inverse Probl. 12, 977 (1996); S. J. Nortan and M. Linzer, IEEE Trans. Biomed. Eng. BME-28, 202 (1981); A. J. Devaney and G. Beylkin, Ultrason. Imaging 6, 181 (1984).

[7] P. M. Morse and K. U. Ingard, Theoretical Acoustics (McGraw-Hill, New York, 1954).

[8] Our time-reversal methods can be extended to an absorbing medium by reversing the absorption coefficient.

[9] P. M. Morse and H. Feshbach, Methods of Theoretic Physics (McGraw-Hill, New York, 1953).

[10] An explicit expression of $g_{1}$, in the form of a series of eigenfunctions, can be found for the boundaries that fit with separable coordinates [9].

[11] First, after combining (13), (17), and the time-domain form of (2), we find that $g_{1}\left(\mathbf{r}, t \mid \mathbf{r}_{d}, t_{0}\right)$ only within $t<A_{g}$, where $A_{g}=t_{0}+\left(\left|\mathbf{r}-\mathbf{r}_{d}\right|+2 l_{\text {obj }}\right) / v_{s}$, is needed to compute $p_{r}\left(\mathbf{r}, \hat{t}_{e}(\mathbf{r})\right)$ using (13) for the DT application. Second, when $\mathbf{r}$ is near the center of $\Sigma$ and $l_{\mathrm{det}}>2 l_{\mathrm{obj}}$, the difference in the flight times of the first two rays (arriving at $\mathbf{r}$ at time $t_{0}+\left|\mathbf{r}-\mathbf{r}_{d}\right| / \boldsymbol{v}_{s}$ ) and the other rays (such as ray $D A B$ ) is larger than $2 l_{\mathrm{obj}} / v_{s}$ in most cases, and, consequently, rays other than the first two make a contribution to $g_{1}\left(\mathbf{r}, t \mid \mathbf{r}_{d}, t_{0}\right)$ only when $t>A_{g}$, and to $p_{r}(\mathbf{r}, t)$ only when $t>\hat{t}_{e}(\mathbf{r})$.

[12] M. A. Fiddy, J. Phys. D 19, 301 (1986). 